\title{
Why Living Kidney Donor Candidates Are Turned Down? A Single-Center Cohort Study
}

Abdulrahman Altheaby ${ }^{1}$, Ahmed Almukhlifi ${ }^{2}$, Abdulrahman Aldoukhi ${ }^{2}$, Abdullah Alfaleh ${ }^{2}$, Ghaleb Aboalsamah $^{1}$, Ala Alshareef ${ }^{1}$, Mohamed Alruwaymi Sr. ${ }^{1}$, Khaled Bin saad $^{1}$, Ziad Arabi ${ }^{3}$

1. Organ Transplant Center and Hepatobiliary Sciences Department, King Abdulaziz Medical City, Riyadh, SAU 2. Medicine, King Saud Bin Abdulaziz University for Health Sciences, Riyadh, SAU 3. Organ Transplant Center and Hepatobiliary Sciences Department, King Abulaziz Medical City, Riyadh, SAU

Corresponding author: Abdulrahman Altheaby, a83sa@hotmail.com

\section{Abstract \\ Introduction}

Living donor kidney transplantation is the best replacement therapy for patients with end-stage renal disease. It offers more benefits than deceased donor transplantation. However, living kidney donors (LKDs) undergo an extensive evaluation to ensure their suitability for donation, and this can result in rejection of many potential donors.

\section{Aim}

The aim of this study was to recognize the reasons for declining LKDs in our Organ Transplant Center at King Abdulaziz Medical City.

\section{Settings and Design}

This was a retrospective study to determine the various reasons to reject an LKD at the Organ Transplant Center.

\section{Methods and Material}

All the LKDs from January 2016 to December 2019 were included. Declined donors were reviewed and data were obtained from the electronic database and transplant nephrology shared files.

\section{Statistical analysis}

We performed data analysis using SPSS version 24.0 (IBM Corp., Armonk, NY, USA). Data for continuous variables were presented as mean \pm standard deviation and were compared using $\mathrm{t}$-test. Categorical variables were presented as frequencies and percentages; chi-square test was used to test for main association and then Bonferroni adjustment was used for post-hoc testing. Statistical significance was considered if a twotailed p-value of $<0.05$ was achieved.

Received 08/05/2020

Review began 08/07/2020 Review ended 08/08/2020 Published 08/19/2020

() Copyright 2020

Altheaby et al. This is an open access article distributed under the terms of the Creative Commons Attribution License CC-BY 4.0., which permits unrestricted use, distribution, and reproduction in any medium, provided the original author and source are credited.

\section{Results}

A total of 410 potential LKDs were evaluated, of whom 241 (58.8\%) successfully underwent donor nephrectomy and 169 (41.2\%) were unable to proceed for kidney donation. The most common reasons for rejection of LKDs were medical (47.9\%) followed by immunological reasons mainly blood group incompatibility (19.5\%). Other reasons were donor withdrawal (15.4\%), recipient-related reasons $(7.1 \%)$, surgically unfit to proceed for nephrectomy (4.7\%), or psychological reasons (2.3\%).

\section{Conclusions}

A significant proportion of potential LKDs did not complete the kidney donation process due to medical, immunological, and surgical reasons. In addition, a proportion of LKDs decided to withdraw at some point during the evaluation process. Investing in donors' educational programs and implementing a standardized evaluation process are essential to increase LKDs pool.

Categories: Internal Medicine, Nephrology, Transplantation

Keywords: living donor, donor evaluation, donor rejection, kidney transplant, transplantation, donor nephrectomy

\section{Introduction}

Renal transplantation is the best treatment option for patients with end-stage renal disease as it significantly improves the patients' quality of life [1,2]. Kidney transplantation is typically classified as deceased-donor (brain death or cadaveric) or living-donor transplantation, which can be either from related 
or non-related donors, depending on the existence of a biological relationship between the donor and recipient. Renal transplantation from living donors offers more benefits than deceased donor transplantation since it is associated with longer graft survival and decreased waiting period on the transplantation list [3]. From 1979 to 2017, a total of 11,509 kidney transplantations were performed in Saudi Arabia, out of which, 7,838 (68.1\%) came from living-related donors, 3,108 (27\%) from deceased donors, and 563 (4.9\%) were from living unrelated donors [4]. However, the number of kidney transplants per year only covers less than $5 \%$ of the dialysis population.

Potential living kidney donors (LKDs) undergo an extensive evaluation to ensure the short- and long-term safety before proceeding for donation [5]. All LKDs must be at least 18 years and have a body mass index (BMI) of less than $35 \mathrm{~kg} / \mathrm{m} 2$, as well as good physical and mental health. In addition, donors should be free from uncontrolled hypertension, diabetes, active malignancy, or active infections [6]. All live kidney donation must be completely a voluntary decision without any pressure or guilt associated with the donation and donors cannot get paid for their donation [7].

By the end, accepting or declining LKDs should depend on whether the benefit outweighs the risk in both the donor and recipient or not. However, there are still considerable variabilities between transplant centers regarding acceptance or rejection of LKDs. Living donors' rejections can be due to medical, surgical, immunological, and/or psychological reasons. In this study, we report our experience with potential LKDs at King Abdulaziz Medical City (KAMC), Riyadh, Saudi Arabia. The main goal is to identify the reasons for declining potential LKDs in our Organ Transplant Center.

\section{Materials And Methods}

All rejected potential LKDs from January 2016 to December 2019 were reviewed and analyzed at the Organ Transplant Center at KAMC. Demographic data, recipient relation, evaluation process, and outcomes (accepted, rejected, withdrawn) were collected using electronic database retrospectively.

The potential LKDs passed through stages of evaluation before being accepted for donor nephrectomy (Figure 1). In the first stage, potential LKDs meet the clinical transplant coordinator, who is responsible for assessing clinical basic and demographic data, as described, as well as taking a detailed history regarding metabolic, cardiac, infectious and renal diseases, and family and psychosocial history. Besides, the coordinator also revises the basic work-up that includes blood group, urine analysis, serum creatinine, and hemoglobin A1c (HbA1c). If the LKD is found to be a drug user, if the decision for donation is made under pressure, or if there is any payment involved with the donation, the LKD is rejected. Otherwise, if there are no contraindications, the evaluation process evolves to the second stage. At this stage, the LKD is submitted to further testing, including 24-hour creatinine clearance, viral serologies (such as HIV), human leukocyte antigens (HLA) tissue typing to perform virtual cross-match against the potential recipient. Subsequently, the potential donor would be interviewed by a nephrologist that reviews, once again, all the work-up, and the LKD is also assessed by a mental health specialist. If there are no clinical contraindications at this stage, the LKD is submitted to a renal CT angiogram (third stage) and evaluated by a transplant surgeon that presents the case to the Transplant Committee for final approval. The LKD may withdraw at any stage of work-up and the reason for that would be confidential. 


\section{Cureus}

Stage 1: Initial Assessment \& Education:

Age , BMI , BP

Medical history and physical examination

Basic work up: Blood Group, Serum Creatinine, HbA1c, Urine Analysis

\section{Stage 2: Specific assessment \& Education \\ Work up: 24 h urine Protein , Creatinine \\ clearance, infectious disease serology, HLA \\ testing , CXR , ECG. Nephrology and mental \\ health assessment.}

\section{Stage 3: Renal CT Volume \\ Transplant surgery and transplant \\ anesthesia assessment}

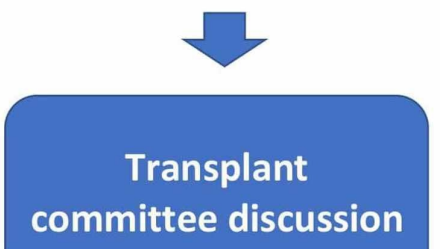

\section{FIGURE 1: Three stages of kidney donors work-up}

BMI, body mass index, BP, blood pressure; HLA, human leukocyte antigen; CXR, chest X-ray; CT, computed tomography; ECG, electrocardiogram

Data analysis was performed using SPSS version 24.0 (IBM Corp., Armonk, NY, USA). Data for continuous variables were presented as mean \pm standard deviation and were compared using t-test. Categorical variables were presented as frequencies and percentages; chi-square test was used to test for main association and then Bonferroni adjustment was used for post-hoc testing. Statistical significance was considered if a twotailed p-value of $<0.05$ was achieved.

\section{Results}

A total of 410 potential LKDs were evaluated at the KAMC Organ Transplant Center from January 2016 to December 2019. Of those, 241 (58.8\%) successfully underwent donor nephrectomy, whereas 169 (41.2\%) were unable to proceed for kidney donation due to different reasons. There was no specific pattern regarding the annual rate of LKDs rejection during the study period, as shown in Table 1. 


\section{Cureus}

\begin{tabular}{|c|c|c|}
\hline Year & Accepted, N (\%) & Declined, N (\%) \\
\hline 2016 & $43(52)$ & $37(48)$ \\
\hline 2017 & $47(53.3)$ & $36(46.7)$ \\
\hline 2018 & 69 (63.9) & $39(36.1)$ \\
\hline 2019 & $96(62.8)$ & $57(37.2)$ \\
\hline
\end{tabular}

TABLE 1: Annual rate of donors revaluation (accepted or declined)

There was no significant difference in accepted and rejected donors' demographics in terms of average age (29 years in accepted vs. 31 years in rejected group) and male predominance (79.6\% in accepted vs. $86.3 \%$ in rejected donors). More than half of the donors were non-smokers in both groups (59.3\% of the accepted group and $54.4 \%$ of the rejected group). Most of LKDs' BMI was between 18.5 and $29.9 \mathrm{~kg} / \mathrm{m}^{2}$ in both groups, although more patients had a BMI of $>30 \mathrm{~kg} / \mathrm{m}^{2}$ in the rejected group versus donated candidates ( $32 \%$ vs. 13.7\%). Regarding kidney function, similar average glomerular filtration rate (GFR) was found in both accepted $\left(113 \mathrm{~mL} / \mathrm{min} / 1.73 \mathrm{~m}^{2}\right)$ and rejected donors $\left(111 \mathrm{~mL} / \mathrm{min} / 1.73 \mathrm{~m}^{2}\right)$, and similar average creatinine $(\mathrm{mg} / \mathrm{dL})$ was found and in accepted $(71.6 \mathrm{mg} / \mathrm{dL})$ and rejected donors $(73.6 \mathrm{mg} / \mathrm{dL})$. HbA1c was also similar, with 5.1 to 5.3 in both, groups and majority of candidates were non-hypertensive in both groups. However, hypertension was more prevalent among rejected LKDs. Table 2 showed the details of demographic data of all evaluated LKDs. 


\section{Cureus}

\begin{tabular}{|c|c|c|c|c|}
\hline & & Successfully completed the donation process & Did not the complete donation process & p-Value \\
\hline \multicolumn{2}{|l|}{ Total, $n=410$} & 241 (58.8\%) & 169 (41.2\%) & \\
\hline \multicolumn{2}{|l|}{ Age (years) } & $31.3 \pm 8.5$ & $32.2 \pm 8.06$ & 0.281 \\
\hline \multirow{2}{*}{ Gender } & Male & $192(79.7 \%)$ & $126(74.6 \%)$ & \multirow{2}{*}{0.221} \\
\hline & Female & $49(20.3 \%)$ & $43(25.4 \%)$ & \\
\hline \multirow{2}{*}{ Hypertension } & Yes & $3(1.2 \%)$ & $12(7.1 \%)$ & \multirow{2}{*}{0.001} \\
\hline & No & 238 (98.8\%) & 157 (92.9\%) & \\
\hline \multirow{2}{*}{ Smoker } & Yes & $98(40.7 \%)$ & 77 (45.6\%) & \multirow{2}{*}{0.323} \\
\hline & No & $143(59.3 \%)$ & 92 (54.4\%) & \\
\hline \multirow{4}{*}{$\mathrm{BMI}\left(\mathrm{kg} / \mathrm{m}^{2}\right)$} & $<18.5$ & $12(5 \%)$ & $7(4.1 \%)$ & \multirow{4}{*}{0.104} \\
\hline & $18.5-24.9$ & $86(35.7 \%)$ & $52(30.7 \%)$ & \\
\hline & $25-29.9$ & $110(45.6 \%)$ & $71(42 \%)$ & \\
\hline & $>30$ & $33(13.7 \%)$ & $39(23 \%)$ & \\
\hline \multirow{4}{*}{ Blood group } & $\mathrm{O}$ & $146(60.6 \%)$ & $123(72.8 \%)$ & \multirow{4}{*}{0.045} \\
\hline & A & $44(18.3 \%)$ & $26(15.4 \%)$ & \\
\hline & B & 48 (19.9\%) & 18 (10.7\%) & \\
\hline & AB & $3(1.2 \%)$ & $2(1.1 \%)$ & \\
\hline \multicolumn{2}{|c|}{ Creatinine $(\mu \mathrm{mol} / \mathrm{L})$} & $71.62 \pm 10.61$ & $73.69 \pm 25.92$ & 0.265 \\
\hline \multicolumn{2}{|c|}{ eGFR (mL/min/1.73 m²) } & $113 \pm 23.34$ & $111.06 \pm 34.12$ & 0.494 \\
\hline \multicolumn{2}{|l|}{ HbA1c } & $5.25 \pm 1.78$ & $5.14 \pm 2.03$ & 0.561 \\
\hline
\end{tabular}

\section{TABLE 2: Demographic data of potential LKDs}

eGFR, estimated glomerular filtration rate

Table 3 demonstrates the relationship of potential donors with the recipients. Most of the donors were related to the recipients, with 192 (79.7\%) in donated LKDs and 110 (65\%) in rejected LKDs. There were more unrelated donors in the rejected group than those who proceeded to donor nephrectomy (59 [35\%] vs. 49 [20.3\%], respectively). The reasons for declining potential LKDs were classified into several subclasses, as shown in Table 4. The most common reason to decline potential LKDs was the presence of medical reasons in 81 (47.9\%) out of 169 rejected LKDs. Medical reasons were divided into several causes outlined in Table 5 and Figure 2. Diabetes mellitus or impaired glucose tolerance, hypertension, and obesity represented almost $50 \%$ of the medical reasons for rejecting LKDs. The second reason for declining potential donors was HLA or $\mathrm{ABO}$ incompatibility, with 33 patients corresponding to $19.5 \%$ of the rejected potential LKDs. Rejection due to surgical reasons such as renal vascular anomalies or double ureter was found in 8 (4.7\%) LKDs. Four LKDs were rejected after mental assessment (2.3\% of the rejected LKDs). A total of 26 (15.4\%) potential LKDs decided to withdraw their consent to donate, of whom 17 (65\%) LKDs were unrelated to recipient. While 12 (7.1\%) LKDs were willing to proceed for kidney donation and appeared good candidates, but they were rejected for recipients' related reasons. In our cohort, there were five (2.9\%) LKDs who were rejected for unknown reasons; we could not identify the reasons, but it seems due to recipient-related issues because their work-up was fine to proceed for donation. 


\section{Cureus}

\begin{tabular}{|l|l|l|l|}
\hline Relation to recipient & Successfully completed donation process, $\mathbf{N}(\%)$ & Did not complete donation, $\mathbf{N}(\%)$ & $\mathbf{p}$-Value \\
\hline Parent & $8(3.3 \%)$ & $6(3.6 \%)$ & $32(18.9 \%)^{\mathrm{a}}$ \\
\hline Offspring & $84(34.9 \%)^{\mathrm{a}}$ & $39(23.1 \%)$ & 0.026 \\
Sibling & $52(21.6 \%)$ & $11(6.5 \%)$ & $8(4.7 \%)$ \\
Spouse & $12(5 \%)$ & $19(11.2 \%)$ \\
Uncle/aunt & $11(4.6 \%)$ & $2(1.2 \%)$ \\
Cousin & $19(7.9 \%)$ & $52(30.8 \%)^{\mathrm{a}}$ \\
Nephew/niece & $6(2.5 \%)$ & \\
\hline Unrelated & $49(20.3 \%)^{\mathrm{a}}$ &
\end{tabular}

\section{TABLE 3: The relationship of potential donor with the recipient}

aSignificant difference was found when column proportions were compared after p-value adjustment using the Bonferroni method.

\begin{tabular}{|l|l|}
\hline \hline Reason & Number (\%) \\
\hline Medical & $81(47.9 \%)$ \\
Immunologic & $33(19.5 \%)$ \\
Surgical & $8(4.7 \%)$ \\
Recipient related & $12(7.1 \%)$ \\
Psychological & $4(2.3 \%)$ \\
Back out & $26(15.4 \%)$ \\
Unknown & $5(2.9 \%)$ \\
\hline TABLE 4: Reasons for declining potential living kidney donors (n = 169) \\
\hline \hline
\end{tabular}




\section{Cureus}

Reason

$\mathbf{N}(\%)=81(47.9)$

Hypertension

9

Diabetes mellitus or impaired glucose tolerance

18

Obesity

11

Proteinuria

5

Hematuria

Renal cysts

8

Renal stone

Low glomerular filtration rate

5

SCA/thalassemia

Tumors

2

2

1

2

Pregnancy

2

TABLE 5: Medical reasons for declining potential living kidney donors

SCA, sickle cell anemia; FL, fatty liver

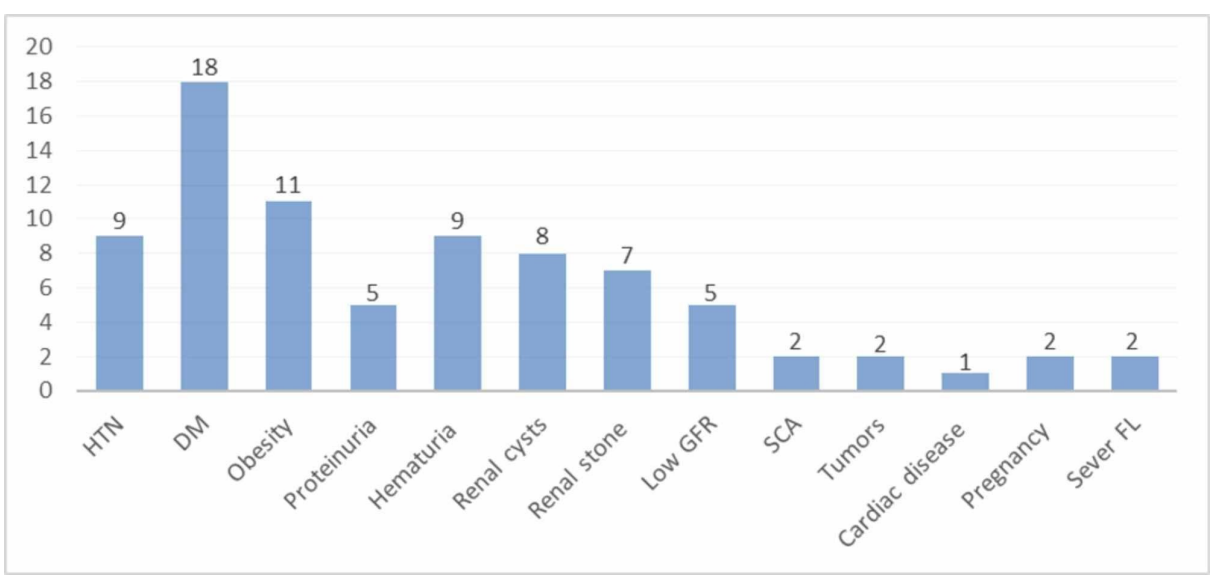

FIGURE 2: Medical reasons for declining potential living kidney donors

HTN, hypertension, DM, diabetes mellitus; GFR, glomerular filtration rate; SCA, sickle cell anemia; Sever FL, severe fatty liver

\section{Discussion}

Living kidney donation contributes to more than $80 \%$ of kidney transplantation in Saudi Arabia [4]. The low rate of deceased donor kidney transplants in Saudi Arabia occurs due to many reasons, of which the most important is related to the cultural background [8]. For this reason, it is essential to expand the living donor pool to meet the rapidly increasing need for organ transplantation in Saudi Arabia. This should be done carefully to ensure living donors and recipient safety. Understating our current practice in LKDs evaluation is very essential to set a protocol to safely expand the LKDs pool.

In our study, more than half (58.8\%) of the evaluated potential LKDs successfully underwent donor nephrectomy, which was similar to another study conducted in New York in which donor nephrectomy was proceeded in (56\%) of their fully evaluated kidney donors [9]. This percentage of donors proceeding for donor nephrectomy was higher when compared to a local study conducted at King Fahad Specialist Hospital 
(KFSH) in Dammam and another study in Turkey in which donor nephrectomy was proceeded only in $32 \%$ and $38 \%$ of their evaluated donors, respectively $[10,11]$.

The majority of the potential donors in our study were young with no significant difference in average age between accepted (29 years) and rejected (31 years) donors, which was similar to the KFSH study where the average age of potential LKDs was 32 years [10]. In contrast, the potential LKDs in our study were younger than LKDs of other studies from western countries where the average age was between 45 to 53 years $[12,13]$. Also, our study showed that both groups were predominantly males (79.6\% vs. $86.3 \%)$. This finding was similar to the KFSH study with a ratio of $3: 1$ (M: F), which could be explained by the cultural background where males usually take the initiative in helping the community [10]. This finding was the opposite to what was found in other studies where female gender predominated [13,14].

Our study also demonstrated that more than half of the donors were non-smokers in both groups (59.3\% and 54.4\%). In general, we advised our donors to stop smoking and offer medical and psychological support to help them quit smoking but we did not decline a donor who is a smoker. In a risk index lately established for LKDs, a history of smoking was a crucial factor in the living donor in comparison to each other and to deceased donor kidneys [15]. Furthermore, the history of cigarette smoking by donors was statistically an important risk factor for both graft and survival of recipients [16].

Regarding the reasons for the rejection of potential LKDs, the most common were medical reasons (47.9\%). Of these, diabetes or impaired glucose tolerance were the most frequent followed by obesity and hypertension. Similarly, other local and global studies showed that the most common reasons for the rejection of potential LKDs were medical with diabetes and hypertension constituting the majority of the medical reasons $[10,11,17]$. At our center, we decline all potential LKDs with diabetes or impaired glucose tolerance based on fasting blood glucose and HbA1c. Furthermore, most of LKDs' BMI was between 18.5 and $29.9 \mathrm{~kg} / \mathrm{m}^{2}$ in both groups, although more patients had a BMI of $>30 \mathrm{~kg} / \mathrm{m}^{2}$ in the rejected group versus donated candidates (32\% vs. 13.7\%). In our program, we accept only LKDs with a BMI of $<30 \mathrm{~kg} / \mathrm{m}^{2}$. Donors with a BMI of $>30 \mathrm{~kg} / \mathrm{m}^{2}$ are counselled to lose weight and encouraged to contact the transplant coordinators when they reach their target weight. Acceptable BMI cutoffs are variable between transplant centers. A survey was conducted by Mandelbrot et al. in 2007 who reported that $50 \%$ of transplant programs use a $35 \mathrm{~kg} / \mathrm{m}^{2}$ limit in the body mass index [18]. Guidelines for KDIGO (Kidney Disease: Improving Global Outcomes) 2017 propose that the decision to accept donor candidates with BMI $>30 \mathrm{~kg} / \mathrm{m}^{2}$ should be taken on a case-by-case basis considering medical conditions and demographic factors [19]. Majority of candidates in our study were non-hypertensive in both groups. Normal blood pressure is appropriate for donation as specified by guidelines for the general population in the country or area where a donation is scheduled. Donor candidates with hypertension that can be controlled with one or two antihypertensive agents to less than 140/90 mmHg and have no evidence of target organ damage could be acceptable for donation [20].

Kidney function evaluation is essential pre-donation. Kidney donors should have normal renal function, without proteinuria or hematuria. In our study, only 5\% LKDs were rejected because of low GFR. In our practice, we follow KDIGO 2012 Chronic Kidney Disease (CKD) guidelines for renal function

evaluations $[21,22]$. GFR of $90 \mathrm{~mL} / \mathrm{min} / 1.73 \mathrm{~m}^{2}$ or greater should be considered an appropriate degree of kidney function for kidney donation, whereas donor candidates with GFR less than $70 \mathrm{~mL} / \mathrm{min} / 1.73 \mathrm{~m}^{2}$ should no longer donate. LKDs with GFR between 70 and $90 \mathrm{~mL} / \mathrm{min}$ would be individualized based on donor age, comorbidity, and other factors. In our study, we found that both groups had similar renal function estimation with almost similar average GFR $\left(\mathrm{mL} / \mathrm{min} / 1.73 \mathrm{~m}^{2}\right)$ of 113 and 111.06 , which was different from an Australian study where $15 \%$ of accepted candidates were found to have a GFR of less than $80 \mathrm{~mL} / \mathrm{min} / 1.73$ $\mathrm{m}^{2}$ [23]. This could be explained by the younger age in our cohort. Overt proteinuria is a contraindication for living donation, whereas persistent microalbuminuria is considered a high risk for donation [24]. In our study, only $5 \%$ of potential LKDs were rejected because of persistent proteinuria and $7 \%$ were rejected due to persistent microscopic hematuria. In general, donor candidates with persistent microscopic hematuria defined as the presence of $>3 \mathrm{RBC} / \mathrm{hpf}$ should have thorough urological evaluation and consideration of a kidney biopsy prior to donation. Persistent hematuria of glomerular origin is a contraindication to living donation because it may indicate renal disease in the donor. However, donors with thin basement membrane disease might be considered in select cases [25]. In our study, kidney biopsies were performed for three donors, of whom one was found to have Alport disease and the other two had IgA (immunoglobulin A) nephropathy. Practices in evaluating and accepting prospective donors with a history of nephrolithiasis vary across transplant centers $[26,18]$. At our center, prospective donors with a history of recurrent unilateral renal stone, bilateral renal stones, or positive metabolic predisposition to stones formation were not accepted for donation. In our study, 7 (8.6\%) potential LKDs were rejected because of nephrolithiasis.

$\mathrm{ABO}, \mathrm{HLA}$, and Rh incompatibility were the second most common reasons for rejection in $19.5 \%$ of the potential LKDs. This is actually contrary to a study conducted in India that showed that blood group incompatibility was the most common reason for rejection (45.8\%) followed by diabetes, renal diseases, and hypertension [27]. In our center, we adopted desensitization protocols, and we are in the process to start paired kidney exchange program to minimize donor rejections due to immunological barriers [28]. 
Declining LKD because of surgical reasons accounted for only $4.7 \%$ of declined donors, although reports were varied from transplant centers. Connaughton et al. found that $13 \%$ of donors declined due to surgical contraindications [12]. The KFSH study showed that $7.9 \%$ of donor rejected for surgical reasons [10]. The main surgical reason to decline an LKD was having complex vasculature, making them unsuitable candidates.

In our study, $15.3 \%$ of evaluated LKDs withdrew their consent to donate at different stages of work-up. This was considered a high percentage as compared to a study conducted in Italy showing that $6.3 \%$ of the reasons of not to proceed for donor nephrectomy were due to donor withdrawal [14]. Almost 30\% of these donors in our study withdrew their consent after completing work-up, which led to a significant waste of resources. Our analysis showed that 17 out of 26 donors who withdrew were unrelated to recipients, although they completed two meetings with the ethical committee for living unrelated donors. This was in agreement with a multivariate analysis by Bailey et al. showed that friend donors were more likely to withdraw from donation [29]. In our cohort, we could not identify clear reasons for donor withdrawal because this information was confidential and not documented in donor records. Studying the reasons for potential LKD withdrawal would allow the transplant programs to improve educational programs and evaluation process to improve living donor recruitment and retention.

This study has several limitations. This is a retrospective, single-center study. The data for this study were obtained by chart review and it was difficult to obtain detailed information that might have influenced the decision of accepting or rejecting the donation. However, despite these limitations, this study reflects our current practice and we need to design a better system to recruit and retain LKDs in an efficient and safe way.

\section{Conclusions}

In conclusion, there is a significant number of potential LKDs that did not proceed for kidney donation. There were different reasons such as medical, immunological, surgical, and consent withdrawal. Working on measures to minimize rejection is essential to meet the transplantation demands. Accepting more marginal donors such as well-controlled hypertension, BMI $30-35 \mathrm{~kg} / \mathrm{m}^{2}$ without other cardiovascular risk factors, adopting paired exchange programs at a national level in Saudi Arabia, and expanding desensitization protocols to overcome immunologic barriers are possible pathways to improve the transplantation rates. In addition, providing more education for LKDs who withdraw and give them more time to come back for donation could increase the LKD pool.

\section{Additional Information \\ Disclosures}

Human subjects: Consent was obtained by all participants in this study. King Abdullah International Medical Research Center (KAIMRC) issued approval RC19/281/R. After reviewing your submitted research proposal/protocol and related documents, the IRB has APPROVED the submission. Animal subjects: All authors have confirmed that this study did not involve animal subjects or tissue. Conflicts of interest: In compliance with the ICMJE uniform disclosure form, all authors declare the following: Payment/services info: All authors have declared that no financial support was received from any organization for the submitted work. Financial relationships: All authors have declared that they have no financial relationships at present or within the previous three years with any organizations that might have an interest in the submitted work. Other relationships: All authors have declared that there are no other relationships or activities that could appear to have influenced the submitted work.

\section{References}

1. Garcia GG, Harden P, Chapman J: The global role of kidney transplantation. J Nephropathol. 2012, 1:69-76. 10.5812/nephropathol.7448

2. Cameron JI, Whiteside C, Katz J, Devins GM: Differences in quality of life across renal replacement therapies: a meta-analytic comparison. Am J Kidney Dis. 2000, 35:629-637. 10.1016/s0272-6386(00)70009-6

3. Nemati E, Einollahi B, Pezeshki ML, Porfarziani V, Fattahi MR: Does kidney transplantation with deceased or living donor affect graft survival?. Nephrourol Mon. 2014, 6:12182. 10.5812/numonthly.12182

4. Annual Report for Organ Transplantation in Kingdom of Saudi Arabia . (2017). Accessed: March 26, 2020: http://www.scot.gov.sa/images/Report_Ar_F_annual\%20report\%202017.pdf.

5. Kasiske BL, Bia MJ: The evaluation and selection of living kidney donors . Am J Kidney Dis. 1995, 26:387-398. 10.1016/0272-6386(95)90664-9

6. Kher A, Mandelorot DA: The living donor evaluation: focus on renal issues . Clin J Am Soc Nephrol. 2012, 7:366-371. 10.2215/CJN.10561011

7. Wright L, Faith K, Richardson R, Grant D: Ethical guidelines for the evaluation of living organ donors . Can J Surg. 2004, 47:408-413.

8. Alghanim SA: Knowledge and attitudes toward organ donation: a community-based study comparing rural and urban populations. Saudi J Kidney Dis Transpl. 2010, 21:23-30.

9. Riehle RA Jr, Steckler R, Naslund EB, Riggio R, Cheigh J, Stubenbord W: Selection criteria for the evaluation of living related renal donors. J Urol. 1990, 144:845-848. 10.1016/s0022-5347(17)39606-4

10. AlBugami MM, AlOtaibe FE, Boqari D, AlAbadi AM, Hamawi K, Bel'eed-Akkari K: Why potential living 
kidney donors do not proceed for donation: a single-center experience. Transplant Proc. 2019, 51:504-508. 10.1016/j.transproceed.2019.01.008

11. Mağden K, Ucar FB, Velioğlu A, Arıkan H, Yeğen ŞC, Tuğlular S, Özener IÇ: Donor contraindications to living kidney donation: a single-center experience. Transplant Proc. 2015, 47:1299-1301. 10.1016/j.transproceed.2015.04.050

12. Connaughton DM, Harmon G, Cooney A, et al.: The Irish living kidney donor program - why potential donors do not proceed to live kidney donation?. Clin Transplant. 2016, 30:17-25. 10.1111/ctr.12641

13. Romagnoli J, Salerno MP, Calia R, Bianchi V, Pedroso JA, Spangoletti G, Citterio F: Expanding the living donor pool, "Ist Act": analysis of the causes of exclusion of potential kidney donors. Transplant Proc. 2013, 45:2632-2634. 10.1016/j.transproceed.2013.08.001

14. Perlis N, Connelly M, D’A Honey JR, Pace KT, Stewart R: Evaluating potential live-renal donors: causes for rejection, deferral and planned procedure type, a single-centre experience. Can Urol Assoc J. 2013, 7:41-45. 10.5489/cuaj. 216

15. Massie AB, Leanza J, Fahmy LM, et al.: A risk index for living donor kidney transplantation . Am J Transplant. 2016, 16:2077-2084. 10.1111/ajt.13709

16. Lin SJ, Koford JK, Baird BC, Hurdle JF, Krikov S, Habib AN, Goldfarb-Rumyantzev AS: Effect of donors' intravenous drug use, cigarette smoking, and alcohol dependence on kidney transplant outcome. Transplantation. 2005, 80:482-486. 10.1097/01.tp.0000168154.14458.28

17. Gozdowska J, Jankowski K, Bieniasz M, et al.: Characteristics of potential living kidney donors and recipients: donor disqualification reasons-experience of a polish center. Transplant Proc. 2013, 45:13471350. 10.1016/j.transproceed.2013.01.030

18. Mandelbrot DA, Pavlakis M, Danovitch GM, et al.: The medical evaluation of living kidney donors: a survey of US transplant centers. Am J Transplant. 2007, 7:2333-2343. 10.1111/j.1600-6143.2007.01932.x

19. Lentine KL, Kasiske BL, Levey AS, et al.: KDIGO clinical practice guideline on the evaluation and care of living kidney donors. Transplantation. 2017, 101:7-105. 10.1097/TP.0000000000001769

20. Lentine KL, Kasiske BL, Levey AS, et al.: Summary of Kidney Disease: Improving Global Outcomes (KDIGO) Clinical Practice Guideline on the evaluation and care of living kidney donors. Transplantation. 2017, 101:1783-1792. 10.1097/TP.0000000000001770

21. CKD Working Group: KDIGO 2012 clinical practice guideline for the evaluation and management of chronic kidney disease. Kidney Int Suppl. 2013, 3:1-150. https://kdigo.org/wpcontent/uploads/2017/02/KDIGO_2012_CKD_GL.pdf

22. Inker LA, Huang N, Levey AS: Strategies for assessing GFR and albuminuria in the living kidney donor evaluation. Curr Transpl Rep. 2017, 4:13-23. 10.1007/s40472-017-0134-0

23. Cash E, Graver A, Kuo S, Kirkland G, Hughes P, Robertson A, Jose MD: Why do not all living kidney donor candidates proceed to donation?. Intern Med J. 2019, 49:384-387. 10.1111/imj.14223

24. Abramowicz D, Cochat P, Claas FH, et al.: European Renal Best Practice Guideline on kidney donor and recipient evaluation and perioperative care. Nephrol Dial Transplant. 2015, 30:1790-1797. 10.1093/ndt/gfu216

25. Kido R, Shibagaki Y, Iwadoh K, Nakajima I, Fuchinoue S, Fujita T, Teraoka S: Persistent glomerular hematuria in living kidney donors confers a risk of progressive kidney disease in donors after heminephrectomy. Am J Transplant. 2010, 10:1597-1604. 10.1111/j.1600-6143.2010.03077.x

26. Alexander RT, Hemmelgarn BR, Wiebe N, et al.: Kidney stones and kidney function loss: a cohort study . BMJ. 2012, 345:5287. 10.1136/bmj.e5287

27. Veerappan I, Neelakantan N, Tamilarasi V, John GT: Medical and non-medical factors that affect voluntary living-related kidney donation: a single-center study. Indian J Nephrology. 2011, 21:14-20. 10.4103/09714065.75223

28. Arabi Z, Theaby A, Farooqui M, Abdalla M, Hajeer A, Abdullah K: The National Guard Health Affairs guidelines for the medical management of renal transplant patients. Saudi J Kidney Dis Transpl. 2018, 29:1452-1469. 10.4103/1319-2442.248311

29. Bailey PK, Tomson CRV, MacNeill S, et al.: A multicenter cohort study of potential living kidney donors provides predictors of living kidney donation and non-donation. Kidney Int. 2017, 92:1249-1260. 10.1016/j.kint.2017.04.020 